\title{
$M^{\mathrm{gr}}$ Albini LeBlanc, deuxième évêque de Gaspé (1946-1957)
}

\section{Paul Joncas}

Volume 46, 1979

URI : https://id.erudit.org/iderudit/1007119ar

DOI : https://doi.org/10.7202/1007119ar

Aller au sommaire du numéro

Éditeur(s)

Les Éditions Historia Ecclesiæ Catholicæ Canadensis Inc.

ISSN

0318-6172 (imprimé)

1927-7067 (numérique)

Découvrir la revue

Citer cet article

Joncas, P. (1979). $\mathrm{M}^{\mathrm{gr}}$ Albini LeBlanc, deuxième évêque de Gaspé (1946-1957).

Sessions d'étude - Société canadienne d'histoire de l'Église catholique, 46,

107-117. https://doi.org/10.7202/1007119ar

Tous droits réservés @ Les Éditions Historia Ecclesiæ Catholicæ Canadensis Inc., 1979
Ce document est protégé par la loi sur le droit d'auteur. L'utilisation des services d’Érudit (y compris la reproduction) est assujettie à sa politique d'utilisation que vous pouvez consulter en ligne.

https://apropos.erudit.org/fr/usagers/politique-dutilisation/ 


\section{Mgr Albini LeBlanc, deuxième évêque de Gaspé (1946-1957)}

$M^{* r}$ Albini LeBlanc, de qui je me propose de vous entretenir, est né à Bouctouche, N.B., le $1^{\text {"r }}$ mai 1894. Il a fait ses études classiques à Caraquet et sa théologie au Grand Séminaire d'Halifax, de 1917 à 1921. Ordonné prêtre par $\mathrm{M}^{\mathrm{rr}}$ Édouard LeBlanc, évêque de St-Jean, N.B., le 15 mai 1921, il fut nommé vicaire à la paroisse Cathédrale, à Moncton, pour devenir ensuite étudiant en théologie à l'Université Angelicum, à Rome. À son retour de Rome, il devint, en 1930, le curé fondatcur de la paroisse Sainte-Thérèse-de-l'EnfantJésus, située près de Moncton. En 1937, il fonda la maison de retraites fermées "Villa St-Joseph», à Bouctouche, qu'il dirigea, tout en y prêchant des retraites.

Le 17 décembre 1940, il est élu évêque de Hearst et ordonné évêque le 11 février 1941, à Moncton, par M $M^{* r}$ Arthur Mćlanson. archevêque de Moncton. Et le 22 décembre 1945, il est nommé évêque de Gaspé et intronisć le 8 mai 1946. Il est décédé accidentellement sur la route à Saint-Majorique, le 17 mai, et a été inhumé le 22 mai 1957.

En s'adressant à ses prêtres, au lendemain de son intronisation comme deuxième évêque de Gaspé, M${ }^{* r}$ Albini LeBlanc leur proposait dans les termes suivants le programme de vie qu'il souhaitait les voir mettre à profit :

Hier soir, je vous ai dit ce que j'entendais être pour vous et pour chacun de vous : " totum singulis".

Laissez-moi vous dire brièvement ce que j’attends de vous, de chacun d'entre vous à titre de prêtre du Christ. Deux choses: que vous soyez des hommes d'étude et des hommes de piété. Étude et piété, science et sainteté : voilà qui résume le prêtre véritable. Deux ailes qui permettent au prêtre de planer au-dessus de l'ignorance religieuse et de la corruption du monde; deux armes qui combattent les ténèbres de l'esprit et la tiédeur du cœur; deux armes nécessaires, car 
la science sans la piété rend le prêtre orgueilleux : la piété sans la science le rend inutile.

J'y reviendrai, et souvent. Je ne vous demanderai pas de faire des merveilles, encore moins des miracles. Mais je vous exhorterai à mieux vous instruire pour éclairer votre peuple : à vous sanctifier pour l'édifier. Et par là, nous obtiendrons tout le reste, c'est-à-dire le zèle des œuvres, des fruits de salut. (Mandements des évêques de Gaspé [MEG]. vol. IV. p. 34.)

Pour avoir été un des collaborateurs immédiats de $M^{*}$ LeBlanc à divers postes de l'administration diocésaine, tout le temps quil fut évêque de Gaspé, je puis affirmer quil demeura, lui, en tout temps, fidèle à lui-même et à son engagement. À ma connaissance. c'est en liaison directe ct continuc avec ces deux pôles, étude ct piété, et alimentéc, nourrie par eux, que s'est élaborée toute l'ceurre pastorale de $\mathrm{M} *$ r LeBlanc.

À telle enseigne que son successeur sur le siège de Gaspí, M $\stackrel{\text { rr }}{ }$ Paul Bernier, pouvait lui aussi au lendemain de son intronisation, le 3 décembre 1957, décrire en ces termes les œuvres et les mérites de ses deux prédécesseurs:

À la Gaspésie en effet, ce Finistère canadien projeté vers l'Atlantique, terre de beauté et d'endurance suil en est. où ladaptation à une âpre nature d'un groupe humain plein de courage et la débordante vitalité dont il fait preuve, constituent un des spectacles les plus émouvants qui se puissent décrire, l'Esprit-Saint a préposé successivement deux Pasteurs. de la taille morale qui fait les géants de la sainteté et de lapostolat : le premier. M*r François-Xavier Ross, véritable pionnier de l'éducation civique et religicuse. apôtre inlassable de la colonisation. et quion a pu appeler avec raison "le libérateur de la Gaspésie ": le second. M"r Albini LeBlanc. admirable de piété. de sagesse et de zèle, qui passera sans doute à l'histoire comme le pourvoyeur inspiré des vocations sacerdotales et religieuses de cette jeunc et florissante Église. (MEG, vol. VII, p. 1)

Je vous convie donc à considérer en $\mathrm{M}^{\mathrm{ar}}$ LeBlanc l'homme $d e^{\prime}$ la prière surtout dans la préparation et la réalisation des Congrès de vocations (1948-1955) de même que dans l'organisation des retraites fermées. Quelques autres ouvres confirmeront. je crois. mon dire. Et je suis d'avis, salvo majori judicio, que le rôle rempli 
par $\mathrm{M}^{\mathrm{gr}}$ LeBlanc en particulier pour sauver, en 1947, le Séminaire de Gaspé, établit de façon indiscutable que l'étude et la science lui importaient souverainement.

Mais au préalable, il convient, ce me semble, de considérer un peu ce qu'était cet homme, même si le rapide portrait que je tenterai d'en tracer ne saurait l'être qu'à larges traits ...

\section{L'HOMME}

On a témoigné avec raison de $\mathrm{M}^{-r}$ LeBlanc que toute son activité fut marquée du signe d'une profonde piété. Je m’en permets ici un exemple. Chaque fois qu'il était dans la possibilité de le faire. il présidait, chaque vendredi soir, dans sa chapelle privée, une heure d'adoration aux intentions de ses diocésains. Il nous en faisait l'annonce au repas du soir et à moins d'empêchement absolument inévitable, nous devions en être, et heureux d'y être. dans lidée de l'Évêque du moins, lui pour qui le prêtre devait être ou du moins prendre les moyens de devenir homme de prière, homme de Dieu. Il faisait oraison pendant près d'une heure avant sa messe. qu il célébrait avec une édifiante piété. J'ajouterais qu'il était l'homme du détail, même méticuleux à certains moments, voire un brin vétilleux. Et disons que c'en était parfois assez agaçant...

On oubliait toutefois ce qui pourrait être appelé ses petites manies - qui n'en a pas? - même ses exigences, fruit de ses convictions et de son austérité personnelles, devant les marques évidentes de bonté qu'il nous prodiguait volontiers. Et nous ne pouvions pas ne pas reconnaître, malgré quelques grimaces ou réticences intérieures. qu'avant de requérir quoi que ce soit des autres, il le vivait d'abord lui-même, le premier ... et qu'il agissait de la sorte parce qu'il aimait vraiment, profondément ses prêtres. Et s'il lui arrivait de leur faire, à l'occasion, une remarque ou l'autre qui s'imposait sur tel ou tel point de leur vie. l'argument surnaturel qu'il employait d'abondance et de source donnait la vraie mesure de sa dilection, tout en entraînant l'adhésion.

Je ne crois pas l'avoir vu ou entendu une seule fois parler en moins bien des autres, surtout de ses prêtres. Et il savait finement détourner la conversation quand nos jeunes ardeurs pouvaient lancer à l'occasion quelques remarques moins obligeantes. 
Et pourtant, $\mathrm{M}^{\mathrm{kr}}$ LeBlanc possédait un tempérament de feu. Il me l'apprit lui-même un jour en avouant tout bonnement qu'il devait lutter fort pour conserver le calme, la patience, la sérénité.

Je voudrais maintenant parler du pasteur que fut, et dans toute la force du terme, $\mathbf{M}^{\mathrm{r} r}$ LeBlanc. Il importe de mentionner dès le départ la richesse de sa parole. Il la proclamait dans un débit facile qu'il savait aisément adapter à ses différents auditoires avec une diction parfaite, un timbre aux intonations absolument justes et qui éclatait comme le clairon. C'était vraiment agréable de l'entendre et de l'écouter.

\section{LES CONGRÈS CATÉCHISTIQUES DES VOCATIONS}

Mais l'œuvre de prédilection de $\mathbf{M}^{\text {ur }}$ LeBlanc aura été sans contredit celle des vocations. Il sut la conserver au cœur même de ses préoccupations pastorales d'évêque, la considérant à bon droit comme absolument essentielle à la vie de l’Église. Il s`en ouvrait à ses prêtres dès le 15 mars 1947 , soit moins d'un an après son arrivée à Gaspé. Je veux noter ici (j'en parlerai plus loin) que c'est également à l'été de cette même année 1947 qu'il eut à assurer la réorganisation et donc la survie du Séminaire diocésain. Il écrit donc à ce moment aux prêtres :

La crise des vocations sacerdotales et religieuses est tristement à l'ordre du jour. À peu près partout. le douloureux soupir du Sauveur fait écho: "La moisson est grande mais les ouvriers sont peu nombreux". Elle se répercute et trouve une inquiétante signification dans les pays encore chrétiens comme dans les contrées où la foi est en baisse... Aujourd'hui, la disette a frappé plus près. Nos diocèses, nos paroisses de chez nous n'ont pas les effectifs voulus. En face de besoins qui se multiplient, de tâches qui s'alourdissent. d'œuvres nouvelles à créer, certains diocèses ne voient se lever qu'une petite troupe d'ouvriers, à peine de quoi remplir les vides...

Les âmes marquées pour le sacerdoce et la vie religieuse, loin d'être une rareté, sont aujourd'hui, comme toujours, très nombreuses. Mais si les vocations se font plus rares, même en nos milieux réputés encore chrétiens, c'est que fait défaut l'esprit surnaturel alimenté et affermi par l'enseignement et les exemples de parents pieux. 
Le premier jardin et le mieux adapté, dit Pie XI, dans l'Encyclique sur le Sacerdoce Catholique, où doivent comme spontanément germer et éclore les fleurs du Sanctuaire, c'est encore et toujours la famille vraiment et profondément chrétienne.

Notre travail en faveur des vocations devra donc en être un de rénovation chrétienne avant tout. Voilà pourquoi nous avons cru que le moyen le plus efficace serait une série de Congrès Catéchistiques de Vocations. (MEG, IV, p. 87ss.)

Il en traçait ensuite les grandes lignes. Cette croisade de prière et d'étude, dirigée et animée par l'Évêque, accapara l'esprit et le cœur du diocèse pendant 6 ans.

Je me souviens de l'occasion où il eut l'inspiration sûrement peu ordinaire de cette formule nouvelle des congrès de vocations. C'était un vendredi soir, après l'heure d'adoration habituelle en sa chapelle privée. Il m'appela à son bureau, où je le trouvai la joie et la détermination inscrites au visage. Il me dit alors qu'une chose l'avait particulièrement frappé, ce soir-là, en méditant sur le texte que nous savons tous : Priez le Maître de la Moisson ... « Nous voulons des prêtres, dit-il, et nous oublions de prier. Eh bien. c'est décidé, nous allons prier publiquement, officiellement, pour obtenir des vocations....»

À compter de cette heure, il s'employa à fond à concrétiser son projet. Il définit d'abord le sens exact de ces corgrès, en précisa les objectifs ; il leur créa une âme, pour ainsi dire, en ces termes :

Il y a deux idées à faire entrer dans les esprits :

1 - chez les parents

a) ils sont les premiers artisans des vocations par leurs vertus et exemples, par l'éducation religieuse des petits commencée de très bonne heure au foyer (à deux ans au plus tard) et à base de catéchisme ;

b) l'école prolonge et complète ce premier travail et, pour cela, doit être catholique.

\section{2 - chez les enfants}

eux, les petits d'aujourd'hui, seront demain les prêtres, les religieux et les religieuses - des paroisses, des écoles, des hôpitaux, des missions, etc... 
Ce thème du Congrès devra déterminer les sujets de prédication des triduums paroissiaux préparatoires au Congrès comme du Congrès lui-même. (Lettre aux PP. du T.S. Sacrement, mars 1947)

Puis il en détermina la formule. Elle s'apparentait certes avec celle bien connue des congrès eucharistiques, qu'organisaient et animaient alors les Pères du T. S. Sacrement. C"est d'ailleurs à ces spécialistes que l'évêque fit appel pour la mise en œuvre de son hardi dessein.

Les Congrès de vocations comportaient cependant des aspects. des points assez particuliers qu'on peut résumer ainsi : chaque congrès était à la fois régional et diocésain. Régional, parce que les paroisses d'un vicariat forain déterminé y prenaient une part immédiate et directe. Diocésain, du fait que toutes et chacune des paroisses du diocèse étaient conviées à préparer le Congrès et à y participer, d'abord par la récitation de la prière spéciale pour les vocations : "Le Congrès se prépare par nos prières et se fera avec nos prières» (MEG, IV, p. 88). Toutes les écoles du diocèse étaient conviées à préparer chacune une exposition catéchistique centrée surtout sur l'Eucharistie et l'Ordre. Sur la campagne de prière si chaudement recommandée par l'Évêque se greffa comme une sorte d'école populaire d'enseignement catéchistique, rappelant aux parents leur rôle irremplaçable dans la culture et l'éclosion des vocations.

Puis, il y avait les triduums des paroisses en congrès, occasions toutes choisies d'intensifier la catéchèse des vocations. Le congrès lui-même débutait le jeudi soir avec l'arrivée au reposoir du cortège marial. C'est ainsi que l'image ou la statue de Notre-Dame du T.S. Sacrement a sillonné les routes du diocèse pendant les six ans qu'ont đuré les congrès, en Gaspésie comme aux Îles-de-la-Madeleine. La Sainte Vierge avait large et pieuse place à chacun des congrès.

La durée du congrès était de trois jours pleins, à compter du jeudi soir. Vendredi, c'était la journée des enfants ; samedi, celle de la famille. Le dimanche, c'était la grande fête des vocations avec ordination à la prêtrise et à d'autres ordres sacrés, une autre particularité de ces congrès. Monseigneur avait obtenu une autorisation spéciale du Pape Pie XII pour présider cette ordination en plein air : chose qui n'était pas permise à l'époque. Je note ici que ce même Pape avait manifesté un vif intérêt à cette formule de prière et d'étude pour les vocations dont $\mathbf{M}^{*}$ r LeBlanc l'avait entretenu. 
lors d'une audience privée, pendant sa visite ad limina, en 1949. Il avait même tenu à parcourir en entier avec lui l'album de photos que $\mathrm{M}^{\mathrm{w} r}$ LeBlanc lui avait offert. À noter qu'à la fin de chaque congrès, une procession aux flambeaux venait couronner le tout en permettant aux congressistes de chanter leur joie.

Au soir du dernier des congrès de vocations, à Saint-Maurice, le 24 juillet $1955, \mathrm{M}^{\mathrm{sr}}$ LeBlanc s'exprimait ainsi, après avoir remercié les artisans de ces assises :

C'est grâce à toutes ces bonnes volontés réunies que ces congrès ont connu un succès non pas seulement extérieur, ce serait trop peu pour les sacrifices qu'ils ont coûté ; mais surtout intérieur. Il y a des âmes qui ont trouvé dans ces congrès, le chemin du retour vers le bon Dieu. Il y a des âmes, et peut-être plus nombreuses encore, qui ont trouvé leur voie et déjà un nombre considérable a pris le chemin des noviciats, des petits et des grands séminaires. Voilà pourquoi je suis très heureux ce soir de rendre un témoignage de reconnaissance profonde à tous nos prêtres et à tous ceux qui ont secondé leurs initiatives.

\section{RÉORGANISATION DU SÉMINAIRE DIOCÉSAIN}

Tout en huilant, si je puis dire, le mécanisme des congrès, $\mathrm{M}^{* \mathrm{r}}$ LeBlanc n'oubliait pas pour autant le problème angoissant que posait, à nouveau, en cette même année 1947, le Séminaire de Gaspé. Les Clercs de Saint-Viateur avaient accepté de prendre la relève à la direction du Séminaire, après le départ des Jésuites en 1938. Il fut question, à plusieurs reprises par la suite, tout au long des années '40, de céder aux Clercs la propriété du Séminaire. Pour diverses raisons, la transaction n'aboutit pas et l'institution demeura diocésaine. Les Clercs en assuraient eux-mêmes l'administration. Malgré leurs efforts, des prodiges d'économie, la terrible dette, cauchemar de $\mathbf{M}^{* r}$ Ross, ne cessait d'augmenter. Je n'oublie pas que, pour aider à en payer les seuls intérêts, un groupe d'étudiants - j'en faisais partie moi-même - avait accepté d'employer les mois de vacances de l'été 1940 à parcourir le diocèse avec une pièce de théâtre. La troupe improvisée en donna 52 représentations...

C'est alors qu'intervint - et il importe, je crois, de le proclamer - la décision sûrement providentielle de $\mathbf{M}^{\mathrm{gr}}$ LeBlanc. 
Intronisé à Gaspé en mai 1946, c'est dès le mois de septembre suivant qu'il attaque de front, avec ses conseillers, la question du Séminaire. Et après mûres délibérations avec les vicaires forains et ses Consulteurs diocésains, il put, le $1^{\cdot r}$ avril 1947, communiquer à ses prêtres la grave décision qui avait été arrêtée : «... Nous en sommes arrivés à la conclusion, écrit-il alors, qu'il fallait consentir tous les sacrifices nécessaires pour assumer la direction totale du Séminaire, et pourvoir, sans délai, à sa réorganisation définitive à tous points de vue. C'est dire que son administration. son enseignement et sa formation seront désormais la responsabilité et la tâche du Clergé séculier » (MEG. vol. IV, p. 94 ). On sait que les Clercs de Saint-Viateur avaient accepté de seconder nos prêtres tout le temps nécessaire, suite à cette réorganisation du Séminaire.

Les prêtres s'attendaient assez à cette décision certes audacieuse de l'Évêque, mais elle les prenait quelque peu à la gorge. Ils se savaient peu nombreux et se demandaient, à bon droit, comment ils pourraient devenir ainsi, de but en blanc, les enseignants que requérait le Séminaire. Mais bientôt la crainte le céda à la sérénité. La décision fut acceptée comme une marque édifiante de foi en la Providence, et tout autant comme une preuve exceptionnelle de confiance de l'Évêque envers ses prêtres. Et ils furent neuf à accepter de relever ce défi.

Il fallait également pourvoir à la question financière. $M^{\text {sr }}$ LeBlanc décréta alors comme obligatoire, parce qu'il n'y avait pas d'autre issue, le versement des trois doliars par famille, naguère demandés par $\mathrm{M}^{\mathrm{wr}}$ Ross, en octobre 1923. Le diocèse comptait alors 14800 familles. Leurs contributions ont donné, de 1947 à 1962, près de $\$ 600000$. En tout, les diocésains auront payé pour leur séminaire : $\$ 710672.05$. Ce sont des chiffres comme ceux-là, d'une éloquence indéniable certes, qui faisaient dire à $\mathbf{M}^{* r}$ Paul Bernier, en 1963 : "Il n'est probablement guère de diocèse canadien dont la population catholique ait contribué aussi généreusement à l'établissement et au maintien d'une institution classique » (MEG, vol. VIII, p. 128). Il faut donc affirmer ici que sans cet énergique coup de barre de $M^{*}$ LeBlanc. le Séminaire n'aurait pas vécu et le CEGEP, qu'on a eu peine à obtenir malgré tout, nous serait passé sous le nez...

$\mathrm{M}^{*}$ LeBlanc a profondément aimé et aidé l'œuvre du Séminaire. Je lui parlais un jour de la cathédrale à construire : "Je l'ai sacrifiée, dit-il, pour le Séminaire : nous verrons plus tard...» 


\section{CHAPELET RADIODIFFUSÉ}

$\mathrm{M}^{\mathrm{gr}}$ LeBlanc fut, je le répète, d'abord Pasteur d'âmes. Son zèle ne pouvait donc se borner aux seules œuvres des congrès et $\mathrm{du}$ Séminaire. C'est lui qui avait réussi, en dépit du problème des distances de la Gaspésie, à organiser la radiodiffusion quotidienne du chapelet, la «Croisade du chapelet ». Il y parvint grâce au concours (très généreux) des autorités du Poste CHNC de NewCarlisle et de la Compagnie de Téléphone Gaspé et Bonaventure, qui ont réglé elles-mêmes tous les détails de l'organisation. L'évêque sut naturellement dire sa joie aux diocésains, le jour même de la première émission, le 4 février 1951: "L'inauguration du 'chapelet radiodiffusé', ce soir, à 7 heures, est un événement qui remplit de joie le cœur de votre évêque, et le vôtre, je n'en doute pas... Nous voulons faire de tout le diocèse une seule grande famille en prière, louant et glorifiant Dieu d'une bouche ct d'un cœur unanimes » (MEG, vol. V, pp. 21-22). Cette émission du 'chapelet en famille' a réussi à vivre pendant 22 ans, soit jusqu'en 1973. Elle rejoignait chaque soir pas moins de 15000 auditeurs en Gaspésie, aux Îlesde-la-Madeleine et au Nouveau-Brunswick.

\section{RETRAITES FERMÉES}

$M^{*}{ }^{* r}$ LeBlanc avait été, avant même de devenir évêque, un ardent apôtre des retraites fermées. Il avait assumé pendant huit ans la direction de la «Villa St-Joseph», maison qu'il avait luimême fondée à Bouctouche, sa paroisse natale. À son arrivéc à Gaspé, en 1946, une maison de retraite existait déjà, à Saint-Edgar, fondéc en 1937 par l'abbé J.-Edgar Miville, alors curé de NewRichmond. Elle était assez exiguë et ne pouvait opérer que pendant la belle saison. Les Oblats de Marie Immaculée en prirent la direction en 1944 et continuèrent l'œuvre au même endroit jusqu'en 1949.

En 1948, fut discutée et décidée la construction de la Maison actuelle de Caps-Noirs. Elle fut inauguréc en 1950, et depuis lors. elle a pu continuer son euvre, résister même à la crise des retraites fermées des années récentes. Elle n'a pas cessé d'accueillir des retraitants venus de tous les coins de la Gaspésie. Monseigneur aimait à dire de cette maison : "Elle n'est pas, dans l'appréciation générale, un monument superflu; mais une clinique indispensable pour donner du sang nouveau à une génération qui tend à s'anémier» $\left(\mathrm{M}^{\sharp r}\right.$ LeBlanc, discours). C'est encore pour parler de cette cuvre que 
$\mathrm{M}^{\mathrm{kr}}$ LeBlanc a rédigé sa dernière lettre circulaire, moins d'un mois avant sa mort. "Le chrétien du monde actuel, écrit-il, est grandement exposé à vivre hors de lui-même, routinier, terne, individualiste. Il a besoin de se plonger, au moins une fois par année, dans la lumière intense, la purification profonde et le remontage si efficace de la retraite fermée » (MEG, vol. VI, p. 218).

$\mathrm{M}^{\mathrm{kr}}$ LeBlanc avait également pensé, et dès son arrivée, à une maison de retraites et de récollections sacerdotales, à Gaspé même. qui devait opérer de façon saisonnière. 11 ne put toutefois réaliser son projet qu'en 1956, en greffant l'œuvre des retraites sur celle du Foyer Notre-Dame, organisée déjà depuis 1953 comme maison pour jeunes filles travaillant à Gaspé. Il inaugura la maison, en mars 1957, soit deux mois avant sa mort, en y prêchant lui-même deux retraites.

\section{AUTRES EUVRES}

D'autres maisons d'enseignement ont retenu l'attention de $\mathrm{M}^{\mathrm{rr}}$ LeBlanc. Je veux mentionner ici, en particulier, l'Académie SaintPierre de Lavernière aux Îles-de-la-Madeleine, dont la situation précaire réclamait aussi une réorganisation. On sait que les îles avaient été détachées de Charlottetown et annexées à Gaspé, le 20 juillet 1946. Mःr LeBlanc pourvut au personnel qui manquait à cette académie en obtenant les services de la Congrégation des Eudistes. C'est en juillet 1948 que cette question fut réglée.

Je note également l'ouverture, en septembre 1947, de l'École Normale de Carleton, sur la recommandation de $\mathrm{M}^{\mathrm{w}}$ LeBlanc. Il importe en outre de rappeler que :

1) c'est grâce à l'intervention énergique et efficace de $M^{*}$ "r LeBlanc auprès des autorités concernées, avec l'appui des évêques LaBrie et Shaeffer, que les Pêcheurs-Unis de Québec ont pu surmonter la grave crise financière qui, à la fin de l'été 1948 , secoua leur organisation au point d'en menacer la survie. En plein désarroi après la démission de ses principaux administrateurs, le Conseil d'Administration de Pêcheurs-Unis avait sollicité l'aide de leur pasteur: "Nous vous prions, lui écrivait le président, de faire tout en votre possible pour que l'œuvre éminemment sociale accomplie par Pêcheurs-Unis de Québec se continue dans la voie que lui avait tracée celui qui en fut le grand bienfaiteur. $M^{* r}$ Ross » (Lettre des Pêcheurs-Unis à M ${ }^{a r}$ LeBlanc). C"était l'époque où on écoutait encore les évêques...; 
2) l'U.P.A., l'U.C.C. de naguère, doit à $M^{\mathrm{kr}}$ LeBlanc de s'être implantée, en 1952, et aussi solidement que l'on sait, en Gaspésie ;

3 ) le Service Social diocésain a été établi par ses soins, en collaboration compétente et étroite de notre confrère Claude Allard, en 1954 ;

4) Caritas-Gaspé, bien connu depuis lors par les cœurs généreux du diocèse, a été lancé par $\mathrm{M}^{\mathrm{ar}}$ LeBlanc, en 1956, pour le soutien des Euvres pastorales diocésaines, et plus spécialement pour celle du Foyer Notre-Dame, acquis en 1953 et agrandi en 1956, qui fut successivement, et parfois en même temps. foyer pour jeunes filles, maison de retraites et qui abrite maintenant des personnes âgées.

Je ne saurais terminer sans noter que $\mathrm{M}^{\mathrm{rr}}$ LeBlanc a subi. pendant sept ans, l'angoissante épreuve de sa vue qui diminuait graduellement. Il avait subi, à l'automne de 1950, mais sans trop de succès, l'opération alors très délicate du décollement de la rétine. Je dois ajouter cependant que ceux qui vivaient auprès de lui ne l'ont jamais entendu en parler pour s'en plaindre. Ce n'est qu'en mars 1957, moins de deux mois avant sa mort. que, pour ma part. je l'entendis me dire, avec une émotion que l'on peut deviner: «Je crains bien de perdre totalement la vue cette année »... Quelle épreuve tout de même, et aussi quelle patience de sa part! Ses activités en furent forcément diminuées, particulièrement au cours des dernières années.

N'empêche que $M^{\text {rr }}$ LeBlanc avait réussi à accomplir. en peu d'années et malgré sa vue "baissante», une œuvre pastorale dont le diocèse aura bénéficié en profondeur...

$\mathrm{M}^{\mathrm{mr}}$ Paul Joncas, v.g..

Évêché de Gaspé. 